\title{
Adenocarcinoma gástrico: nuevas opciones terapéuticas
}

P. Khosravi Shahi, G. Pérez Manga. Adenocarcinoma gástrico: nuevas opciones terapéuticas. An Med Interna (Madrid) 2007; 24: 107-108.

La incidencia global del cáncer gástrico ha disminuido en los países industrializados en las últimas décadas. Aunque continua siendo la segunda causa de muerte por cáncer en el mundo, por detrás del cáncer del pulmón (1). Las diferencias geográficas en la incidencia del cáncer gástrico no se conoce bien, pero más de la mitad de los casos se dan en Japón y China, y se ha relacionado con algunos factores de riesgo como dietas ricas en salados y ahumados, y pobres en vegetales y frutas, infección por Helicobacter pylori, anemia perniciosa, tabaquismo, y antecedentes familiares (2).

Se ha producido un cambio en el patrón de presentación del cáncer gástrico en los países occidentales, de manera que la incidencia relativa de los cánceres gástricos proximales, localizados en cardias y unión gastro-esofágica, ha ido en aumento; mientras que la incidencia del cáncer gástrico distal (fundus y antro) va decreciendo progresivamente. Estos dos tipos de cáncer gástrico presentan una historia natural y un comportamiento biológico muy diferentes, así los cánceres gástricos proximales son de un comportamiento clínico mucho más agresivo, con desarrollo precoz de metástasis a distancia por vía hematógena; mientras que los distales suelen diseminarse de forma locorregional antes de dar metástasis hematógenas.

La supervivencia del cáncer gástrico depende sobre todo del estadio de la enfermedad al diagnóstico, de manera que en los estadios más localizados (adenocarcinoma gástrico precoz) la supervivencia global (SG) a los 5 años es de 70-95\% tras la cirugía radical, mientras que la supervivencia mediana en los casos metastáticos sin tratamiento es de aproximadamente 3-4 meses. Sin embargo, el pronóstico del cáncer gástrico en general es infausto, ya que la mayoría de ellos se diagnostican en fases avanzadas.

En los casos operables la cirugía radical continua siendo el único tratamiento curativo, siendo la gastrectomía subtotal/total con linfadenectomía D1 (extirpación de todos los ganglios perigástricos, sin esplenectomía, ni pancreatectomía distal) el tratamiento quirúrgico de elección en los países occidentales. Sin embargo, en Japón donde la incidencia de cáncer gástrico es mucho más elevado, y cuyos cirujanos han desarrollado una gran experiencia, la extensión de la linfade- nectomía de elección es mayor, realizando de forma electiva una resección D2 o incluso una ampliación hacia los ganglios paraaórticos, sin que ello suponga una mayor morbi-mortalidad perioperatoria, consiguiendo supervivencias de dos a tres veces superior (3).

No obstante, a pesar de la cirugía radical la probabilidad de recidiva locorregional y a distancia es elevada. Por ello, es necesario la consolidación con un tratamiento complementario en un intento de erradicar la enfermedad microscópica residual. En la actualidad disponemos de dos alternativas terapéuticas (quimiorradioterapia concomitante coadyuvante y quimioterapia perioperatoria) que han demostrado claramente en sendos ensayos clínicos fase III su eficacia con respecto a la cirugía sola. Varios meta-análisis han evidenciado el escaso beneficio de la quimioterapia adyuvante tras la cirugía radical. En dos de ellos hubo un beneficio mínimo en la supervivencia, que no conllevaron cambios en la práctica clínica (4-6). Un ensayo randomizado demostró un aumento significativo pero pequeño en la supervivencia con la radioterapia sola preoperatoria en pacientes con cáncer de cardias (7).

En la mayoría de los países occidentales el tratamiento estándar del cáncer gástrico resecable continua siendo la cirugía radical seguido de quimiorradioterapia concomitante, basándose en los resultados del ensayo fase III llevado a cabo por Mac Donald y cols. (8). En este estudio con una mediana de seguimiento de 5 años la mediana de SG fue de 36 meses en la rama de la quimiorradioterapia concomitante, y de 27 meses en la rama de la cirugía sola $(\mathrm{p}=0,005)$.

El estudio MAGIC, llevado a cabo por Cunningham y cols. (9), es un ensayo aleatorizado fase III, que incluía 503 pacientes con cáncer gastro-esofágico no metastático, en el que se comparó la cirugía radical sola frente a la quimioterapia pre y postoperatoria con esquema ECF (Epirrubicina, Cisplatino y 5-Fluoruracilo). En este estudio se demostró un aumento estadísticamente significativo de la supervivencia libre de progresión (SLP), con "hazard ratio" (HR) para la progresión de 0,66 (intervalo de confianza al 95\% [IC95\%]: $0,53 ; 0,81 ; \mathrm{p}<0,001)$ a favor de la quimioterapia perioperatoria. Asimismo, este estudio demostró una SG a los 5 años significativamente mayor para la rama de ECF (36 vs. 23\%; 
$\mathrm{p}=0,009)$, con un HR para la muerte de 0,75 (IC95\%: 0,60; 0,93). Es decir, la quimioterapia perioperatoria con ECF disminuía el riesgo de muerte en un $25 \%$ con respecto a la cirugía sola. Los resultados de este estudio ha llevado a que en la actualidad se considere la quimioterapia perioperatoria como una alternativa terapéutica a la quimiorradioterapia.

En el tratamiento de la enfermedad metastática también han aparecido novedades de interés como la superioridad del regimen DCF (Docetaxel, Cisplatino y 5-Fluoruracilo) sobre el esquema estándar en Estados Unidos CF (Cisplatino y 5Fluoruracilo). El estudio V325, llevado a cabo por Cutsem EV y cols. (10), evidenció un aumento significativo del tiempo hasta la progresión ([THP] 5.6 meses para la rama de DCF y 3,7 meses para la rama de CF; p < 0,001) y de la SG en la rama de DCF (9,2 vs. 8,6 meses, respectivamente; $\mathrm{p}<$ 0,02).

Numerosos ensayos fase II han puesto de manifiesto la utilidad de diversos agentes quimioterápicos en el tratamiento de la enfermedad metastática, tales como irinotecan, oxaliplatino, bevacizumab, cetuximab o capecitabina.

\section{Bibliografía}

1. Jemal A, Tiwari RC, Murray T, et al. Cancer statistics, 2004. CA Cancer J Clin 2004; 54: 8-11.

2. Kurtz RC, Sherlock P. The diagnosis of gastric cancer. Semin Oncol 1985; 12: 11-8.

3. Maruyama K, Okabayashi K, Kinoshita T. Progress in gastric cancer surgery in Japan and its limits of radicality. World J Surg 1987; 11: 418-425.

4. Hermans J, Bonenkamp JJ, Boon MC, et al. Adjuvant therapy after curative resection for gastric cancer: meta-analysis of randomized trials. J Clin Oncol 1993; 11: 1441-7.

5. Earle CC, Maroun JA. Adjuvant chemotherapy after curative resection for gastric cancer in non-Asian patients: revisiting a meta-analysis of randomized trials.Eur J Cancer 1999; 35: 1059-64.

6. Mari E, Floriani I, Tinazzi A, et al. Efficacy of adjuvant chemotherapy after curative resection for gastric cancer: a metaanalysis of published randomised trials- a study of the GISCAD (Gruppo Italiano per lo Studio dei Carcinomi dell'Apparato Digerente). Ann Oncol 2000; 11: $837-43$.

7. Zhang ZX, Gu XZ, Yin WB, Huang GJ, Zhang DW, Zhang RG. Randomized clinical trial on the combination of preoperative irradiation and surgery in the treatment of adenocarcinoma of gastric cardia
El ensayo REAL-2, cuyos resultados preliminares han sido publicados en el prestigioso congreso de la ASCO 2006, demuestra la equivalencia en la sustitución de Oxaliplatino y Capecitabina por Cisplatino y Fluoruracilo en el regimen ECF $(11,12)$. En este estudio no hubo diferencias estadísticamente significativas en la tasa de respuestas, ni en la SLP entre las 4 ramas. En la comparación de las ramas de platinos (oxaliplatino y cisplatino), la mediana de SG fue de 10,4 meses para el oxaliplatino frente a 10,0 meses de la rama del cisplatino; y en la comparación de las ramas de fluoropirimidinas, la mediana de SG fue de 9,6 meses para la rama de fluoruracilo y de 10,9 meses para la capecitabina.

\section{P. KHOSRAVI SHAHI, G. PÉREZ MANGA}

\author{
Servicio de Oncología Médica. Hospital General \\ Universitario Gregorio Marañón. Madrid
}

(AGC)—report on 370 patients. Int J Radiat Oncol Biol Phys 1998; 42: 929-34.

8. Macdonald JS, Smalley SR, Bendetti J, et al. Chemoradiotherapy after surgery compared with surgery alone for adenocarcinoma of the stomach or gastroesophageal junction. N Engl J Med 2001; 345: 725-30.

9. Cunningham D, Allum WH, Stenning SP, et al. Perioperative chemotherapy versus surgery alone for resectable gastroesophageal cancer. $\mathrm{N}$ Engl J Med 2006; 355: 11-20.

10. Cutsem EV, Moiseyenko VM,Tjulandin S, et al. Phase III Study of Docetaxel and Cisplatin Plus Fluorouracil Compared With Cisplatin and Fluorouracil As First-Line Therapy for Advanced Gastric Cancer: A Report of the V325 Study Group.J Clin Oncol 2006; 24: 4991-4997.

11. Sumpter KA, Harper-Wynne C, Cunningham D, et al. Report of two protocol planned interim analyses in a randomized multicentre phase III study comparing capecitabine with f luorouracil and oxaliplatin with cisplatin in patients with advanced esophagogastric cancer receiving ECF. Br J Cancer 2005; 92: 1976-83.

12. Cunningham D, Rao S, Starling $\mathrm{N}$ et al. Randomized multicentre phase III study comparing capecitabine with fluorouracil and oxaliplatin with cisplatin in patients with advanced oesophagogastric cancer: The REAL-2 trial. J Clin Oncol 2006; 42: 182. 